\title{
GMI EFFECT SENSING ON MAGNETIC MICROWIRES
}

\author{
Josef BLAŽEK, Pavol LIPOVSKÝ, Miroslav ŠMELKO, Katarína DRAGANOVÁ*, Ladislav \\ TOMČO \\ Technical University of Košice, Faculty of Aeronautics, Rampová 7, 04121 Košice, Slovakia \\ Pavel RIPKA \\ Department of Measurement, Faculty of Electrical Engineering, Czech Technical University in \\ Prague, Technicka 2, Prague 7, Czech Republic \\ *Corresponding author. E-mail: katarina.draganova@tuke.sk
}

\begin{abstract}
One of the perspective methods for the magnetic field measurement is the methodology based on the GMI effect measurement. The improvement of the sensor is in term of the sensor sensitivity and the wide-band measurement range in comparison to the commercially available magnetometers. For the evaluation of the impedance of the sample the lock-in amplifier capable to make a decomposition of the impedance to the real and imaginary part was used. The designed and constructed measurement workstation can be used for the precise impedance measurement. Specification of the related metrological properties of the samples improve the precision of the magnetic field measurement that is important for vehicles navigation or for precise mapping of the magnetic fields if unmanned aerial vehicles are used in non-destructive archaeology.
\end{abstract}

Keywords: GMI effect; microwire; lock-in amplifier; contact

\section{INTRODUCTION}

Nowadays the issue of the magnetic measurements is a widely discussed topic. There are many types of magnetic sensors that have been developed. The measurement of the magnetic field has a wide range of application possibilities not only in the aviation but also in many industrial, technical and also personal applications [1,2].

\section{GMI EFFECT}

The GMI effect is characterized by the change of the complex impedance of the sample excited by the high-frequency current depending on the external magnetic field. The GMI effect sensing is a contact measurement method, which can be used for the microwire characteristics analysis [3]. From the information about the current magnitude flowing through the sample $i_{A C}$ and about the voltage magnitude across the sample $u_{A C}$ we get the value of the impedance module for the given magnetic field. The phase rations between the current and voltage carry information about the magnitude of the real and imaginary part of the impedance.

The measured microwire is connected to the AC power supply and the voltage drop and electrical current are measured (Fig. 1). The external excitation field is generated by DC source and excitation coil. For the GMI measurements, the frequency in the range of $\mathrm{kHz}$ up to the $\mathrm{GHz}$ is used. Nowadays technologies allow manufacturing of many microwire types with different chemical compositions of the core, with different diameters and also with the different ratios of the glass and the core. The glasscoated microwire used as a magnetometer probe can have also different length, various contacting methods can be used, postprocessing methods can be different and also the various defects caused during the manufacturing process have to be taken into consideration. The differences among the microwire samples lead to the necessity to design an automatic measurement workstation which is 
able to measure the fundamental sample characteristics as a result of the impedance change due to the influence of the magnetic field.

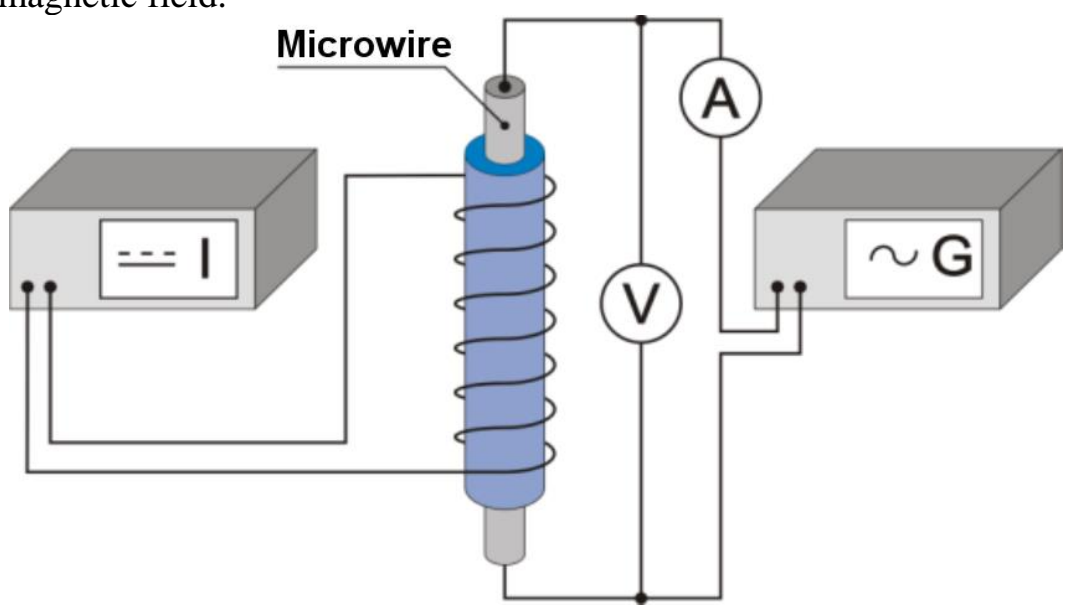

Figure 1 Principle schematics of the GMI effect measurement

\section{LOCK-IN AMPLIFIER}

The lock-in amplifiers can be used for the detection and measurement of very small alternating signals which can achieve the magnitudes of only several $\mathrm{nV}$. The precise measurement can be performed for the measurement of very noisy signals, the information part of which can be thousand times smaller than the noise. The lock-in amplifiers use the principle known as the phase and frequency-sensitive detection, which refers to the determination of the signal component with the specific reference frequency and phase. Other signals with the different frequency are suppressed and have no significant influence on the measurement.

The architecture of the lock-in amplifiers can be changed due to the specific requirements on the evaluation of the measured signal but the principle of the amplifier remains the same. The measured signal which will be evaluated is led to the input of the lock-in amplifier. The first active block is the low-noise inputs amplifier providing the voltage amplification of the signal. The band pass filter for the noise elimination on the other frequencies is often used. The next block is the mixer or phasesensitive detector which multiplies the amplified and filtered input signal with the reference signal. Convenient synchronization of the measured and stimulation signals is necessary to achieve the same value of the frequency and phase. The output of the mixer is created by the summed and subtracted signal of the input and reference signals.

The information value is carried by the direct component of the output signal; therefore, it can be extracted by the low-pass filter with a suitable limiting frequency. The block scheme of the measurement system using an analogue lock-in amplifier is shown in the Fig. 2. The lock-in amplifier is divided into several functional blocks. As it can be seen, the lock-in amplifiers consists of two main parts, which were also designed and realized on two separate printed circuit boards. 


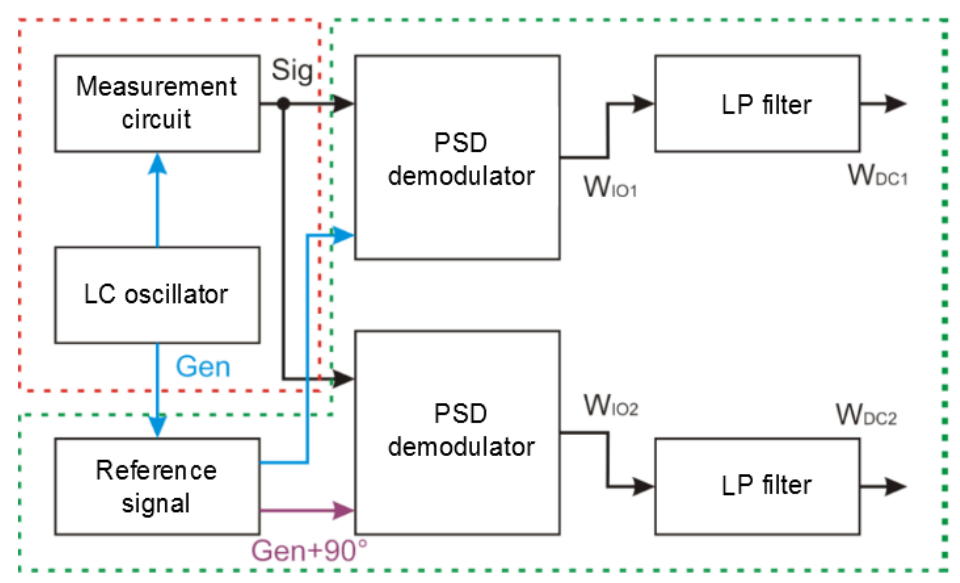

Figure 2 Block scheme of the lock-in amplifier

\section{GMI EFFECT MEASUREMENT WORKSTATION}

The necessity of the automated measuring process of the GMI effect led to the construction of the specialized measurement workstation (Fig. 3) [4]. The first part of this workstation is created by the excitation circuit for the magnetic field generation. This part involves the current source used as the voltage-current converter and the excitation coil. The second part is the evaluation circuit with the designed and realized lock-in amplifier, the output of which is connected to the $\mathrm{A} / \mathrm{D}$ converter of the computer.

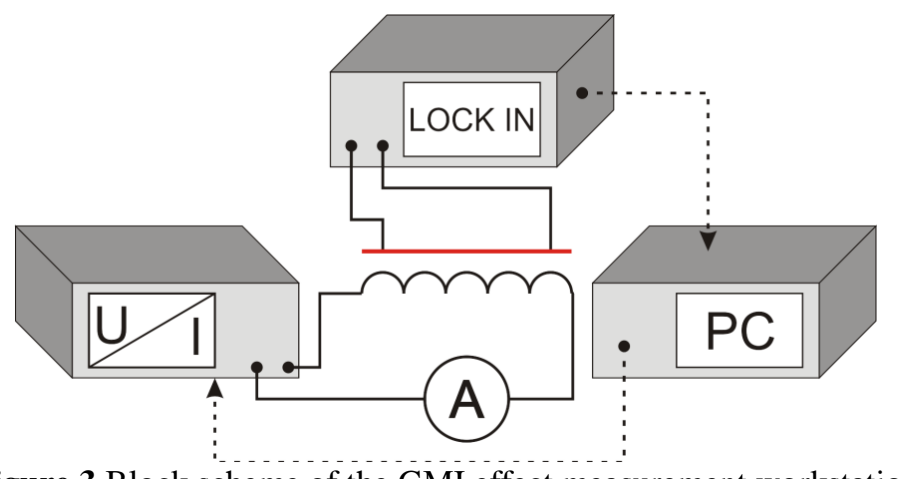

Figure 3 Block scheme of the GMI effect measurement workstation

The homogeneous magnetic field generation is the first task that had to be solved. As the magnetic field generator the coils are used. The mechanical construction of these coils can be different according to the specific application. Both types of coils can be seen in the Fig. 4. The first applied type of the coil was a solenoid - direct long round coil with the turns being wounded narrowly to each other. The homogeneous magnetic field in this case is created in the geometrical axis of the solenoid. The proposed and constructed solenoid was designed for the generation of high magnetic fields. Its diameter is $86 \mathrm{~mm}$ because of the fact that the sample has to be placed into the coil together with the lock-in amplifier. The sample has to be placed into the geometrical centre of the coil as accurately as possible. However, the current source limits allow exciting the coil using the current of only $2 \mathrm{~A}$. This electrical current creates the magnetic field with the intensity of $9 \mathrm{kA} / \mathrm{m}$. For the application it was a satisfactory value. The homogeneity of the magnetic field generated in the solenoid was also convenient, especially if shorter samples were measured. The area with the homogeneous intensity was approx. $2.5 \mathrm{~cm}$. If more precise measurements in weaker magnetic fields are performed, the homogeneity error along the sample has to be considered. 

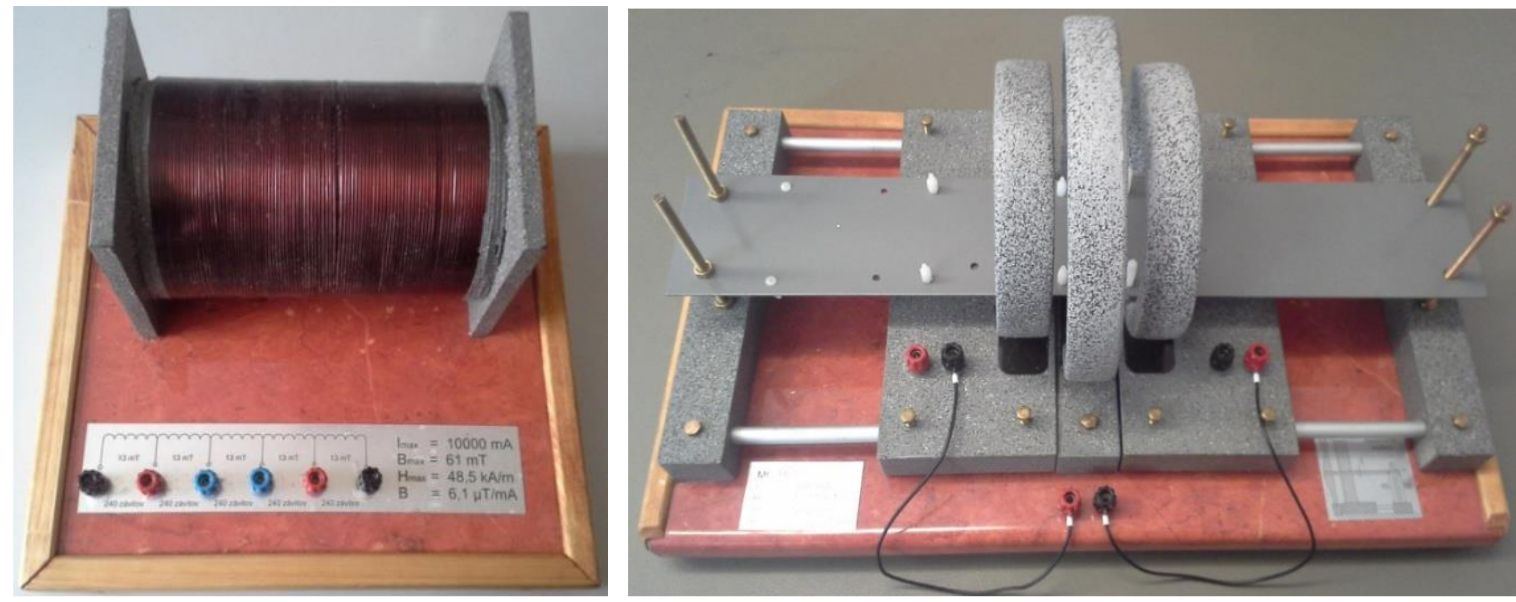

Figure 4 Coils for the magnetic field generation - solenoid (left) and modular coil (right)

The solenoid coil limits pointed out to the necessity of a more complex coil construction, with the magnetic field intensity of $1.2 \mathrm{kA} / \mathrm{m}$ and with the larger length in comparison to the solenoid. Therefore, the coil system construction was designed with the larger homogeneous area. These coils can be arranged as Helmholtz or Maxwell coils in the system of narrow solenoids with a high diameter with a precisely defined diameter, width, distance and number of turns of every coil from the system. For this application the coil system marked as MC 15.1. was created. It is a modular arrangement of three coils where the coil in the middle is static and the other two coils are adjustable and allow modification of the magnetic field by their distance or current flowing through the coils. The second arrangement is characterized by the large homogeneous area of the magnetic field intensity in regard to the dimensions. However, this arrangement has also higher construction demandingness. The final arrangement was able to generate magnetic fields up to the $1200 \mathrm{~A} / \mathrm{m}$. The homogeneous area of the magnetic field intensity achieves $70 \mathrm{~mm}$ in the length. Therefore, the Maxwell coil system is designed for the creation of weak and precise magnetic fields in larger area. The measurement of the microwire characteristics is in this case advantageous also because of the fact that the positioning of the sample will not result in the additional measurement error.

\section{CONTACTING}

Creation of a conductive connection of a high quality between the metal core of a microwire and the measuring equipment, which can be performed with a good repeatability, is one of the most important steps by the contact measurement methods of the microwire characteristics.

At first sight a very simple task of contacting is due to the microwire dimensions significantly complicated. The contacting itself can be divided into several steps. For the creation of a conductive connection on the microwire it is necessary to remove the glass cover from the part of the microwire, which will be contacted. This step is certainly necessary only for the glass-coated microwires. For the removal of the glass cover mechanical and chemical methods were used. The mechanical removal of the glass coat is a simple and quick method for the creation of the place for the conductive connection on the microwire (Fig. 5). The methodology is based on the application of the mechanical force on the microwire coat until it is not broken and separated from the metal core of the microwire. 


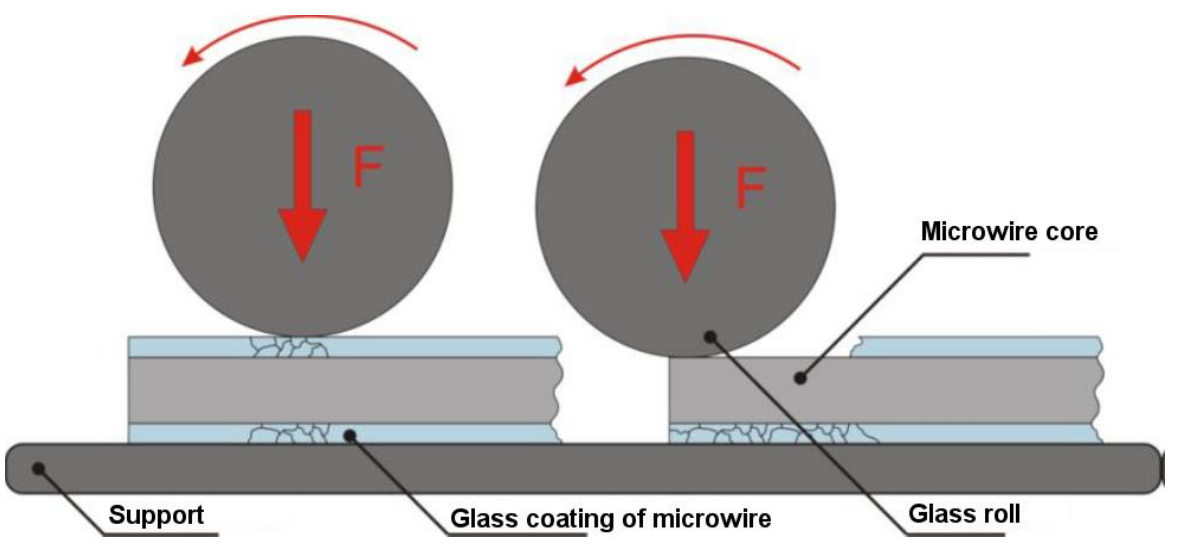

Figure 5 Methodology for the mechanical removal of the glass coat from the microwire

However, it was found out that applying this methodology it is not possible to remove the glass coat with a good reliability and the samples do not have the same characteristics [5]. Although the part of the microwire without the glass coat seems to be homogeneous without the remnants of the glass, the technology itself influences also the metal core of the microwire. Therefore, the technology is convenient only for microwires with larger diameters where for the metal core deformation higher force is necessary. During the mechanical method application also the remaining glass coat of the microwire can be split. These defects together with the ambiguity in the quality of the process were the main cause for the change of the methodology.

The most convenient seems to be the method based on the chemical removal of the glass coating. For this method the acid was used. The manufacturing of these samples was based on the chemical removal of the precisely defined parts of the microwire sample. The initial attempts pointed out to the necessity to cover the remaining part of the sample covered with glass because the glass etching process runs not only in the acid but also in its vapours. As the cover the wax was used.

The microwire was than exposed to the acid for 30 minutes. The contact between the metal core and copper contacts on the PCB was created using the connective adhesives. There are many commercially available types of conductive adhesives. Already the initial attempts with the contacting of the microwire samples pointed out to the fact that not every contact adhesive is convenient for the microwire contacting.

The conductive adhesives are manufactured on the basis of conventional adhesive mixed with the conductive metal powder. Creation of the connection between the contact and a microwire, which does not have precisely defined properties that causes a measurement error and the application of these samples is therefore not possible. For the contacting multiple conductive adhesives were used (Fig. 6). The measured adhesives were characterized by the high liquidity, their adhesion to the contact was good and only in few samples there was a problem with the conductivity.
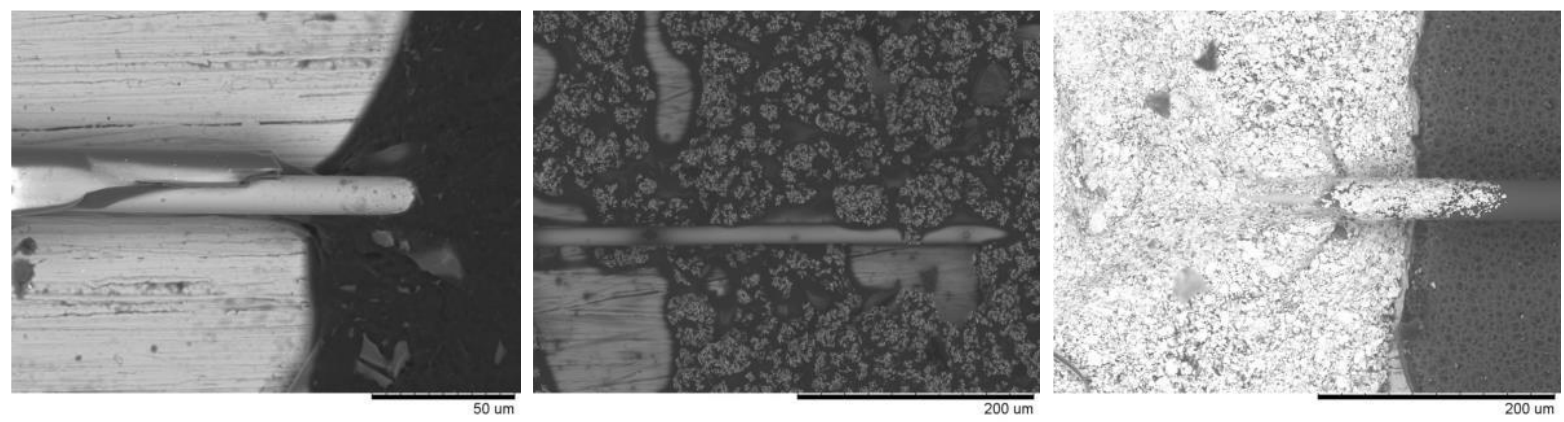

Figure 6 Contact created using the conductive adhesives 
Soldering using a soft solder is a generally used methodology in electronics. The created connection most often between two copper components is permanent and resistant against corrosion and mechanical failures. The soldering itself is performed using the soft tin solder with the temperature of $300{ }^{\circ} \mathrm{C}$. If the copper components are soldered, on the copper surfaces due to the temperature and connection of the copper and tin, the bronze is produced. The chemical connection of these elements is the reason of the quality of the created connection.

However, during the microwire soldering a conductor with a complex chemical composition has to be soldered to the copper part of the PCB. Therefore, it is questionable how the soldered contact between the microwire and copper surface will be created. Only detailed analysis has shown that the quality of the resulting contact is influenced by the fact that the tin solder is not adhesive to the microwire core. The resulting contact cannot be therefore considered for a full-value soldered contact. In this case a sort of a mechanical contact is created (Fig. 7). The tin solder creates in the proximity of the metal coil a tight tube, in which a microwire is placed. The microwire tends to have a longbow shape due to the induced mechanical stresses during the manufacturing and therefore it produces pressure on this tube created in the tin solder. This interaction creates a mechanical contact.

The improvement of the soldered contact can be achieved in several ways. The first one is the utilization of the soldering lotions improving the soldering process. The first tested was LETOL. It did not have a significant influence on the quality of the contact. The second methodology for the improvement of the process was applying the metallization of the microwire sample using a suitable solderable metal. The most convenient way is the sample copper coating. The copper coating of the metal components can be realized using different technologies. However, the resulting effect is the same weather galvanic or chemical copper coating is used. Obviously for our application the chemical copper coating was used.
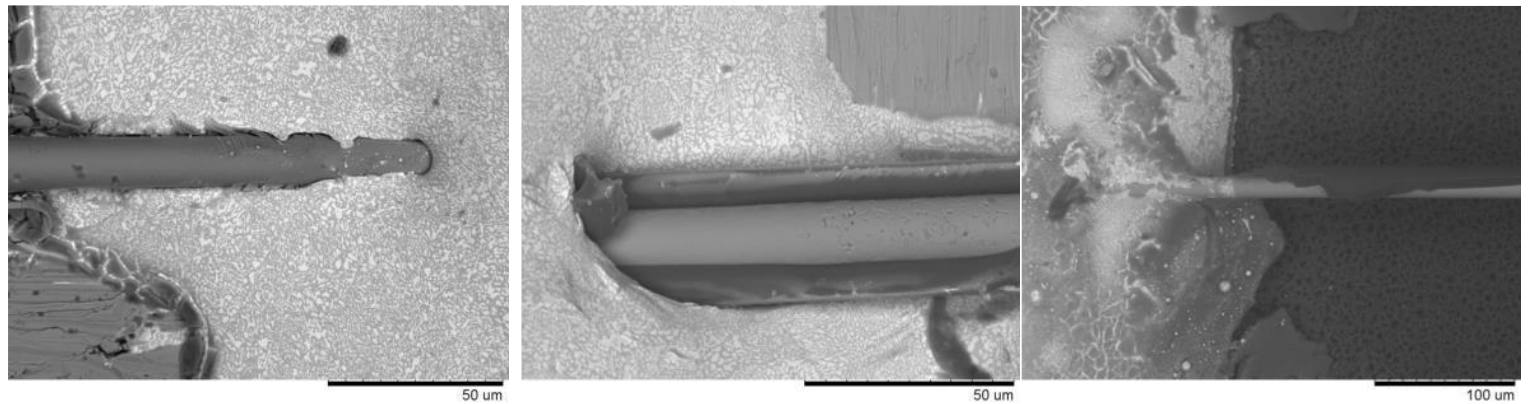

Figure 7 Soldered contact of a microwire sample

During the process of copper coating in the solution the atom from the microwire is replaced by the copper atom in the grid. This technology requires only the immersion of the component into the prepared solution. The coppering solution is based on the copper sulphate and sulphur acid. For the improvement of the resulting surface of the copper-coated sample the adhesive manufactured by the Surtec was added.

The tin solder itself accreted to the microwire sample also during the short time intervals during the soldering. The tin adjoined to the microwire core and the resulting contact was not only mechanical but also chemical because we can suppose creation of the bronze layer between the solder and microwire. The chemical metallization can be performed for any microwire type. The main groups of the microwires are based on the ferrous or cobalt and in these cases it is possible to replace these atoms by copper.

The quality of the conductivity and also the mechanical strength, stability and corrosion resistance predetermine this type of contacting in the top of the methods that can be realized. The disadvantage of this methodology can be the influence of the heating on the sample. The temperature influence on the GMI effect was not precisely defined because of the missing knowledge about the temperature, time and spatial distribution of the heat in the sample. After the performed experiments in the area of quality of the microwire contacting using the conductive glues or soldering the most convenient 
solutions were recommended. Also important is the fact that before the soft solder utilization it is convenient to metallize the sample using for example copper coating.

\section{MEASUREMENTS AND RESULTS}

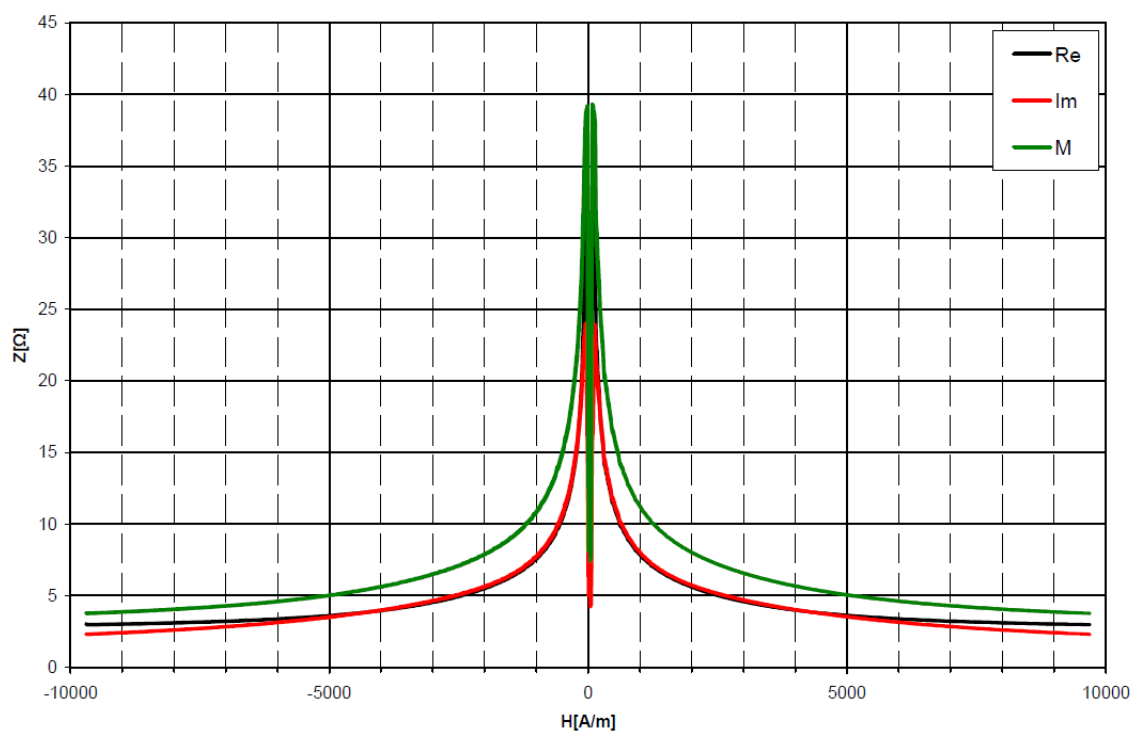

Figure 8 Example of a sample impedance dependence on the magnetic field in the range of $\pm 9 \mathrm{kA} / \mathrm{m}[6]$

The correct settings and conversion of measured value was done by PC card. The precisely defined values of the driving current flowing through the drive coil allow us creation of the magnetic fields with a precise value. Recording the value of the real and imaginary components of the impedance allows us to generate dependencies of sample impedance to a magnetic field, which is shown in Fig. 8. Such automatic measurement stand allows us to quickly and easily determine the size of GMI effect and define approximate characteristics that might be used in the GMI effect based magnetometers.

\section{CONCLUSION}

GMI effect measurement is one of the perspective methods used for the magnetic field measurement. The improvement of the GMI effect measurement on the magnetic microwires was achieved using the designed and constructed workstation for the evaluation of the impedance of the sample the lock-in amplifier capable to make a decomposition of the impedance to the real and imaginary part. For the measurement workstation construction also different methods of contacting were analysed and the most convenient methods were recommended.

\section{Acknowledgement}

This work was supported by the project No. 019/2019/1.1.3/OPVaI/DP with the ITMS code 313011 T557 entitled Support of the research and development potential in the area of transportation vehicles.

\section{References}

[1] D. Praslička, J. Blažek, J. Hudák, I. Mikita, V. Moucha. Industrial applications of magnetometry. JEEEC (2015), Vol. 66, No. 7s, p. 190-192.

[2] P. Ripka, Magnetic Sensors and Magnetometers. DOI: 10.1088/0957-0233/13/4/707. Artech House Publisher, Boston, London, 2001. 
[3] Komová, E. Amorphous and Nanocrystalline Glass-Coated Microwires for Sensor Applications. New Trends in Aviation Development 2014: proceedings of the 11. international scientific conference, Kosice: TU, 2014, p. 1-3. ISBN 9788055316505.

[4] Kán, V., Šmelko, M., Lipovský, P. Meracie pracovisko GMI efektu založené na princípe LOCK IN zosilňovača. Senzorika a magnetometria 2016. Košice: TU, 2016. P. 6-11. ISBN 978-80-5533051-8.

[5] Šmelko, M., Kravčák, J., Praslička, D., Blažek, J., Draganová, K. Impact of modified endings on noise characteristics of Fe-based glass-coated microwires. Journal of Electrical Engineering, 2015. Bratislava: STU, 2015, Vol. 66, No. 7/s, p. 30-32. ISSN 13353632.

[6] Kán, V., Bajús, J., Volčko, T., Kliment, T., Flachbart, N. Testing of GMI Effect on Modern Magnetic Materials. 5th International Scientific Conference of Ph.D. Students and Young Scientists and Researchers. Košice: TU, 2016. P. 1-6. ISBN 978-80-553-2514-9.

Received 11, 2019, accepted 12,2019

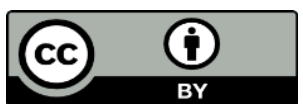

Article is licensed under a Creative Commons Attribution 4.0 International License 